\title{
Inhibition Determines Membrane Potential Dynamics and Controls Action Potential Generation in Awake and Sleeping Cat Cortex
}

\author{
Michelle Rudolph, ${ }^{1}$ Martin Pospischil, ${ }^{1}$ Igor Timofeev, ${ }^{2}$ and Alain Destexhe ${ }^{1}$ \\ ${ }^{1}$ Integrative and Computational Neuroscience Unit, Centre National de la Recherche Scientifique, 91198 Gif-sur-Yvette, France, and ${ }^{2}$ Department of \\ Anatomy and Physiology, Laval University, Québec, Canada G1K7P4
}

\begin{abstract}
Intracellular recordings of cortical neurons in awake cat and monkey show a depolarized state, sustained firing, and intense subthreshold synaptic activity. It is not known what conductance dynamics underlie such activity and how neurons process information in such highly stochastic states. Here, we combine intracellular recordings in awake and naturally sleeping cats with computational models to investigate subthreshold dynamics of conductances and how conductance dynamics determine spiking activity. We show that during both wakefulness and the "up-states" of natural slow-wave sleep, membrane-potential activity stems from a diversity of combinations of excitatory and inhibitory synaptic conductances, with dominant inhibition in most of the cases. Inhibition also provides the largest contribution to membrane potential fluctuations. Computational models predict that in such inhibition-dominant states, spikes are preferentially evoked by a drop of inhibitory conductance, and that its signature is a transient drop of membrane conductance before the spike. This pattern of conductance change is indeed observed in estimates of spike-triggered averages of synaptic conductances during wakefulness and slow-wave sleep up states. These results show that activated states are defined by diverse combinations of excitatory and inhibitory conductances with pronounced inhibition, and that the dynamics of inhibition is particularly effective on spiking, suggesting an important role for inhibitory processes in both conscious and unconscious cortical states.
\end{abstract}

Key words: intracellular recordings; conductance analysis; cerebral cortex; computational models; GABAergic conductances; spiketriggered average

\section{Introduction}

Intracellular recordings of cortical neurons in awake, conscious cats and monkeys show a depolarized membrane potential $\left(V_{\mathrm{m}}\right)$, sustained firing, and intense subthreshold synaptic activity (Matsumura et al., 1988; Baranyi et al., 1993; Steriade et al., 2001; Timofeev et al., 2001). It is presently unclear how neurons process information in such active and irregular states. An important step to investigate this problem is to obtain a precise characterization of the conductance variations during activated electroencephalogram (EEG) states. Input-resistance measurements indicate that during such activated states, cortical neurons can experience periods of high conductance, which may have significant consequences for their integrative properties (Destexhe and Paré, 1999; Kuhn et al., 2004) (for review, see Destexhe et al., 2003). In anesthetized animals, several studies have provided

Received 0ct. 26, 2006; revised March 6, 2007; accepted March 22, 2007.

This work was supported by Centre National de la Recherche Scientifique, Agence Nationale de la Recherche, the Human Frontier Science Program, and the European Community (A.D.), and the Canadian Institutes of Health Research (CIHR) and the Natural Sciences and Engineering Research Council of Canada (I.T.). I.T. is a scholar of the CIHR. We dedicate this paper to the memory of Mircea Steriade, and we are very grateful to him for many stimulating discussions and continuous support.

Correspondence should be addressed to Alain Destexhe, Integrative and Computational Neuroscience Unit, Centre National de la Recherche Scientifique, 91198 Gif-sur-Yvette, France. E-mail: destexhe@iaf.cnrs-gif.fr.

DOI:10.1523/JNEUROSCI.4652-06.2007

Copyright $\odot 2007$ Society for Neuroscience $\quad$ 0270-6474/07/275280-11\$15.00/0 measurements of the excitatory and inhibitory conductance contributions to the state of the membrane, using various paradigms (Borg-Graham et al., 1998; Hirsch et al., 1998; Paré et al., 1998; Anderson et al., 2000; Wehr and Zador, 2003; Priebe and Ferster, 2005; Haider et al., 2006). However, no such conductance measurements have been made so far in awake and naturally sleeping animals.

In the present study, we provide such estimates using a combination of intracellular recordings and computational models. Intracellular recordings were performed in the association cortex of nonanesthetized cats across wake or sleep states, according to a technique described previously (Steriade et al., 2001; Timofeev et al., 2001). Intracellularly recorded neurons display a highly complex and irregular subthreshold synaptic activity from which we estimate the conductances (and their variances) by matching stochastic models to the intracellular data. We also use the same approach to estimate the time course of relative conductance changes during the transition between depolarized and hyperpolarized phases (up and down states), as seen intracellularly during slow-wave sleep (SWS). We next introduce computational models with stochastic conductances reproducing the above measurements to infer the type of conductance variations that underlie spiking activity in such states. Parts of this paper have been published previously in abstract form (Destexhe et al., 2005; Pospischil et al., 2005). 


\section{Materials and Methods}

Intracellular recordings in awake and naturally sleeping animals. Intracellular recordings were performed in cat association cortex (areas 5, 7, and 21 of the parietal cortex) using a technique described in detail previously (Steriade et al., 2001; Timofeev et al., 2001). Briefly, in a chamber specially designed for chronic intracellular recordings, local field potential (LFP), electromyogram (EMG), and electrooculogram (EOG) electrodes as well as head fixation screws were implanted under anesthesia. Electrodes for LFP recordings were inserted in cortical depth and their tips were located at a depth of $0.8-1.0 \mathrm{~mm}$. The animals were gradually habituated to the recording setup and the head-fixed position. After 3-4 $\mathrm{d}$ of training, the cats started to display normal sleep-waking cycles and could move their limbs.

Intracellular recordings were performed under current clamp using standard borosilicate glass micropipettes (outer diameter, $1.5 \mathrm{~mm}$; inner diameter, $0.84 \mathrm{~mm}$ ) filled with $2.5 \mathrm{~mm}$ potassium acetate. Initially, pipettes were pulled to a resistance of $120-150 \mathrm{M} \Omega$ and under visual guidance their tips were broken to obtain a resistance of 50-70 $\mathrm{M} \Omega$. The pipettes descended in the recording chamber. After insertion in the brain, the resistance of some of the pipettes further decreased. The intracellular electrodes with resistance lower than $30 \mathrm{M} \Omega$ were discarded because intracellular recordings obtained with these electrodes often revealed signs of deterioration appearing as unstable membrane potential, which becomes progressively depolarized, and broadening of action potential duration. Typically, the recording sessions lasted up to $3 \mathrm{~h}$. Only recordings showing stable $V_{\mathrm{m}}$ (no drift) and overshooting action potentials are considered here. To correctly estimate the membrane potential, we recorded the potential drop during penetration of the recorded neuron and after pulling out of the neuron. If the difference in the estimation of recorded membrane potential at the beginning and at the end of recording exceeded $2 \mathrm{mV}$, these data were discarded. A total of 118 neurons were recorded during the wake/sleep cycle, including 96 presumed excitatory cells and 22 presumed inhibitory interneurons (see Results).

To accumulate sufficient statistics for the analysis (see below), recordings needed to last typically $>30 \mathrm{~min}$ and be conducted using several levels of injected different holding currents (DCs). A total of 15 neurons satisfying these criteria were obtained and were used for conductance analysis. In some cases, the recording was long enough to span across several states of vigilance [quiet wakefulness, slow-wave sleep, and rapid eye movement (REM) sleep]. Experiments were conducted in agreement with ethics guidelines of the Canadian Council on Animal Care and were approved by the committee for animal care of Laval University.

Analysis and computational models. The basis of the analysis was to match the $V_{\mathrm{m}}$ of the intracellularly recorded neurons to a stochastic model of synaptic background activity. This model was used for estimating synaptic conductances and to perform spike-triggered averages of synaptic conductance variations related to spikes, as well as for simulating membrane dynamics based on those estimates. The methods related to these different approaches are described below.

The basis of the analysis was a "fluctuating conductance model," which approximates the highly complex synaptic activity as a random process, using global synaptic conductances described by the following stochastic equation (Destexhe et al., 2001):

$$
\frac{d g_{e}(t)}{d t}=-\frac{1}{\tau_{e}}\left[g_{e}(t)-g_{e 0}\right]+\sqrt{\frac{2 \sigma_{e}^{2}}{\tau_{e}}} \xi_{e}(t),
$$

where $g_{e}(t)$ is the global excitatory conductance, fluctuating around the mean value $g_{e 0}$ and with variance $\sigma_{e}^{2}, \tau_{e}$ is the excitatory time constant, and $\xi_{e}(t)$ is a Gaussian white noise source with zero mean and unit SD. The global inhibitory conductance $g_{i}(t)$ is described by an equivalent equation with parameters $g_{i 0}, \sigma_{i}^{2}, \tau_{i}$ and noise source $\xi_{i}(t)$. These conductances determine the subthreshold variations of the membrane potential $V(t)$, according to the following membrane equation:

$$
C \frac{d V(t)}{d t}=-G_{\mathrm{L}}\left[V(t)-E_{\mathrm{L}}\right]-g_{e}(t)\left[V(t)-E_{e}\right]-
$$

$$
g_{i}(t)\left[V(t)-E_{i}\right]+I_{\mathrm{ext}},
$$

where $C$ denotes the membrane capacitance, $I_{\text {ext }}$ a stimulation current, $G_{L}$ the leak conductance, and $E_{L}$ the leak reversal potential, whereas $E_{e}$ and $E_{i}$ are the reversal potentials of $g_{e}(t)$ and $g_{i}(t)$, respectively.

The advantage of this stochastic model is that different analytic approximations are available for the steady-state $V_{\mathrm{m}}$ distribution $(\mathrm{VmD})$. The $\mathrm{VmD}$ method consists of matching this analytic expression to $V_{\mathrm{m}}$ distributions obtained experimentally, leading to estimates of excitatory and inhibitory conductances, as well as their variance, solely from current-clamp recordings (for a detailed discussion and testing of this method, see Rudolph et al., 2004). The method is based on the assumption of Gaussian distribution for synaptic conductances. The validity of this approximation was tested in numerical simulations (Destexhe et al., 2001; Rudolph and Destexhe, 2003, 2005) and theoretical investigations (Rudolph and Destexhe, 2006), as well as experimental studies (Rudolph et al., 2004, 2005). $V_{\mathrm{m}}$ distributions must be obtained at least at two DC levels, and using multiple current levels allows one to identify the linear range of the voltage-current $(V-I)$ relationships (see Fig. $3 A$ ). This is an important check to limit the possible contamination of subthreshold voltage-dependent conductances. To insure that only subthreshold activity is used for the analysis, action potentials, if present, were removed by excluding all $V_{\mathrm{m}}$ activity within a window of $5 \mathrm{~ms}$ before and $10 \mathrm{~ms}$ after the spike. To avoid the possible bias caused by removing compound EPSPs before spikes, the $\mathrm{VmD}$ estimates were performed in hyperpolarized segments with little spiking activity; the testing of this method using dynamic-clamp experiments indicates that such a bias is negligible $(\mathrm{Ru}-$ dolph et al., 2004).

Because two DC levels are necessary to estimate conductances, $n>2$ levels provides multiple estimates corresponding to different ranges of $V_{\mathrm{m}}$. Up to $n=8$ current levels were used here, which leads up to 28 possible pairings. For each pairing, the fitting of the $V_{\mathrm{m}}$ distributions with the analytic expression [in particular its Gaussian approximation (Rudolph et al., 2004)] yields estimates of the synaptic conductances and their variances, provided a number of other parameters are kept fixed. These parameters are the synaptic reversal potentials $\left(E_{e}=0 \mathrm{mV}\right.$ and $E_{i}$ $=-75 \mathrm{mV})$ and the decay time constants $\left(\tau_{e}=3 \mathrm{~ms}\right.$ and $\left.\tau_{i}=10 \mathrm{~ms}\right)$ of synaptic conductances, as well as the leak conductance $\left(G_{\mathrm{L}}\right)$ (see Results) and reversal potential $\left(E_{\mathrm{L}}=-80 \mathrm{mV}\right)$. The values of $\tau_{e}=3 \mathrm{~ms}$ and $\tau_{i}=$ $10 \mathrm{~ms}$ were obtained from the power spectra of $V_{\mathrm{m}}$ activity (Rudolph et al., 2005). The leak parameters were estimated based on previous intracellular experiments realized on neurons of the same area of cat cortex (area 5-7) after application of tetrodotoxin (Paré et al., 1998): after suppression of network activity, the membrane conductance was reduced by approximately five times, and the resting $V_{\mathrm{m}}$ was of $-80 \mathrm{mV}$ on average. The "optimal values" of conductance presented here (see Fig. $3 C$ ) were based on the assumption that the up states of these experiments (ketamine-xylazine anesthesia) have the same relative impact on the membrane as the up states of natural slow-wave sleep analyzed here. This assumption is supported by the fact that the absolute values of input resistance during up states are similar in the two preparations (Paré et a., 1998; Steriade et al., 2001). We performed variations around this estimate (see Results) to check that the results obtained do not qualitatively depend on this assumption. Note that the values of the conductance variances are not affected by the particular choice of the leak conductances (Rudolph et al., 2004) (see Results).

We also performed a spike-triggered average (STA) conductance analysis from $V_{\mathrm{m}}$ activity. The average conductance time course related to spikes was estimated using a method outlined previously, and which was tested in real neurons with dynamic clamp (Pospischil et al., 2007). The STA of the voltage is calculated first, and the method searches for the "most likely" spike-related conductance time courses $\left[g_{e}(t), g_{i}(t)\right]$ that are compatible with the observed voltage STA. This estimation is made by discretizing the time axis in Equations 1 and 2, which leads to a discretized system from which one can evaluate the probability distribution for the spike-related conductance time courses. Because this distribution is symmetric, the STAs of the synaptic conductances can be determined by maximizing its probability distribution, which amounts to differentiating the distribution and subsequently solving a system of algebraic equations (Pospischil et al., 2007). The system of algebraic equations is solved using standard numerical methods (Press et al., 1986). 
To quantify the conductance STA, we fitted the conductance time course using the exponential template

$$
g_{e}(t)=g_{e 0}\left(1+k_{e} \exp \left(\frac{t-t_{0}}{T_{e}}\right)\right)
$$

for excitation, and an equivalent equation for inhibition. Here, $t_{0}$ stands for the time of the spike, $k_{e}$ quantifies the maximal increase/decrease of conductance before the spike, with time constant $T_{e}$ (and similarly for inhibition). We also calculated the "relative conductance change" before the spike:

$$
r_{g}=\frac{g_{e 0} k_{e}-g_{i 0} k_{i}}{g_{e 0}+g_{i 0}} .
$$

Here, the terms $g_{e 0} k_{e}$ and $g_{i 0} k_{i}$ quantify the absolute excitatory and inhibitory conductance change before the spike, respectively. The difference between these two contributions is normalized to the total synaptic conductance. A negative value indicates an overall drop of total membrane conductance before the spike (see Fig. 9A), whereas a positive value indicates an increase of total conductance (supplemental Fig. 4, available at www.jneurosci.org as supplemental material).

To relate the STA analysis to the $\mathrm{VmD}$ analysis, we quantified the "relative excess conductance" by calculating the quantity:

$$
e_{g}=\frac{g_{e 0}-g_{i 0}}{g_{e 0}+g_{i 0}} \text {. }
$$

Here, a negative value indicates a membrane dominated by inhibitory conductance, whereas a positive value indicates dominant excitatory conductance.

Similarly, we calculated the "relative excess conductance fluctuations" by evaluating the quantity:

$$
s_{g}=\frac{\sigma_{e}-\sigma_{i}}{g_{e 0}+g_{i 0}} .
$$

Finally, the fluctuating conductance model (Eq. 2) was simulated numerically together with voltage-dependent $\mathrm{Na}^{+}$and $\mathrm{K}^{+}$conductances responsible for generating action potentials [Hodgkin-Huxley type models with parameters identical as those described by Destexhe et al., (2001)]. The conductance values obtained from intracellular data were integrated in this model to verify that the $V_{\mathrm{m}}$ activity and firing behavior obtained match the recordings of the corresponding cell. Simulations were performed on Linux workstations using the Neuron simulation environment (Hines and Carnevale, 1997).

\section{Results}

Intracellular recordings in awake and naturally sleeping animals

Intracellular recordings of cortical neurons were performed in parietal cortex of awake and naturally sleeping cats (see Materials and Methods). Intracellular recordings were done simultaneously with the LFP, EMG, and EOG to identify behavioral

$(\mathrm{Hz})$
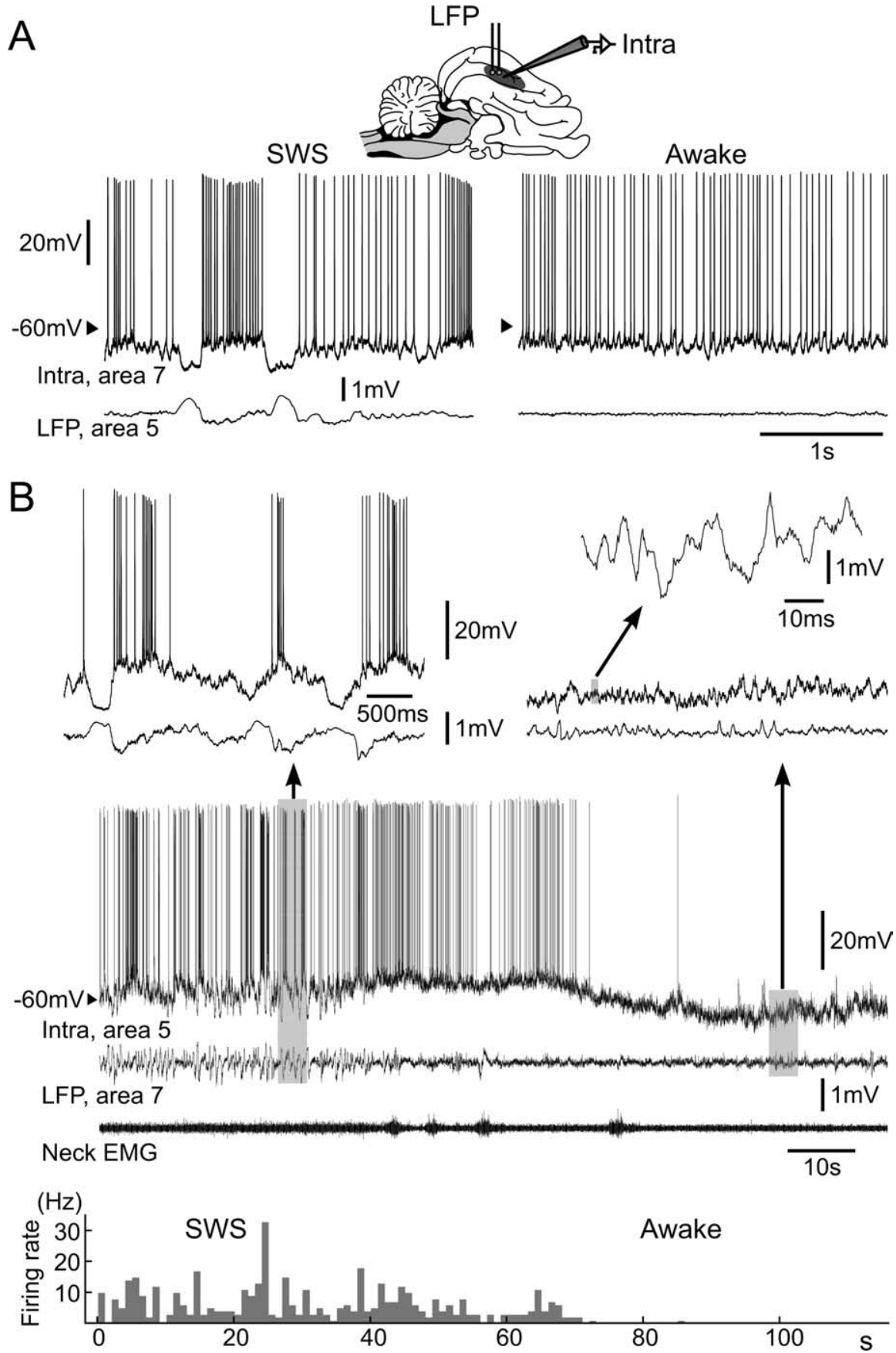

Figure 1. Activity of regular-spiking neurons during slow-wave sleep and wakefulness. $A$, Wake-active regular-spiking neuron recorded simultaneously with LFPs (diagram) during SWS and wakefulness (awake) condition. $\boldsymbol{B}$, Wake-silent regular-spiking neuron recorded simultaneously with LFPs and EMG during slow-wave sleep to wake transition. Slow-wave sleep was characterized by high-amplitude low-frequency field potentials, cyclic hyperpolarizations, and stable muscle tone (expanded in the top left). Low-amplitude and high-frequency fluctuations of field potentials and muscle tone with periodic contractions characterized the waking state. This neuron was depolarized and fired spikes during initial $30 \mathrm{~s}$ of waking, then hyperpolarized spontaneously and stopped firing. A fragment of spontaneous $V_{m}$ oscillations is expanded in the top right. A period with barrages of hyperpolarizing potentials is further expanded as indicated by the arrow.

states. With pipettes filled with $\mathrm{K}^{+}$-acetate, we recorded and electrophysiologically identified activities of 96 presumed excitatory neurons during the waking state. Of them, 47 neurons revealed a regular-spiking (RS) firing pattern, with significant spikefrequency adaptation in response to depolarizing current pulses, and spike width of $0.69 \pm 0.20 \mathrm{~ms}$ (range, $0.4-1.5 \mathrm{~ms}$ ). The $V_{\mathrm{m}}$ of RS neurons varied in a range between -56 and $-76 \mathrm{mV}$ (mean, $-64.0 \pm 5.9 \mathrm{mV}$ ). Twenty-six of these RS neurons were "wake- 
A SWS

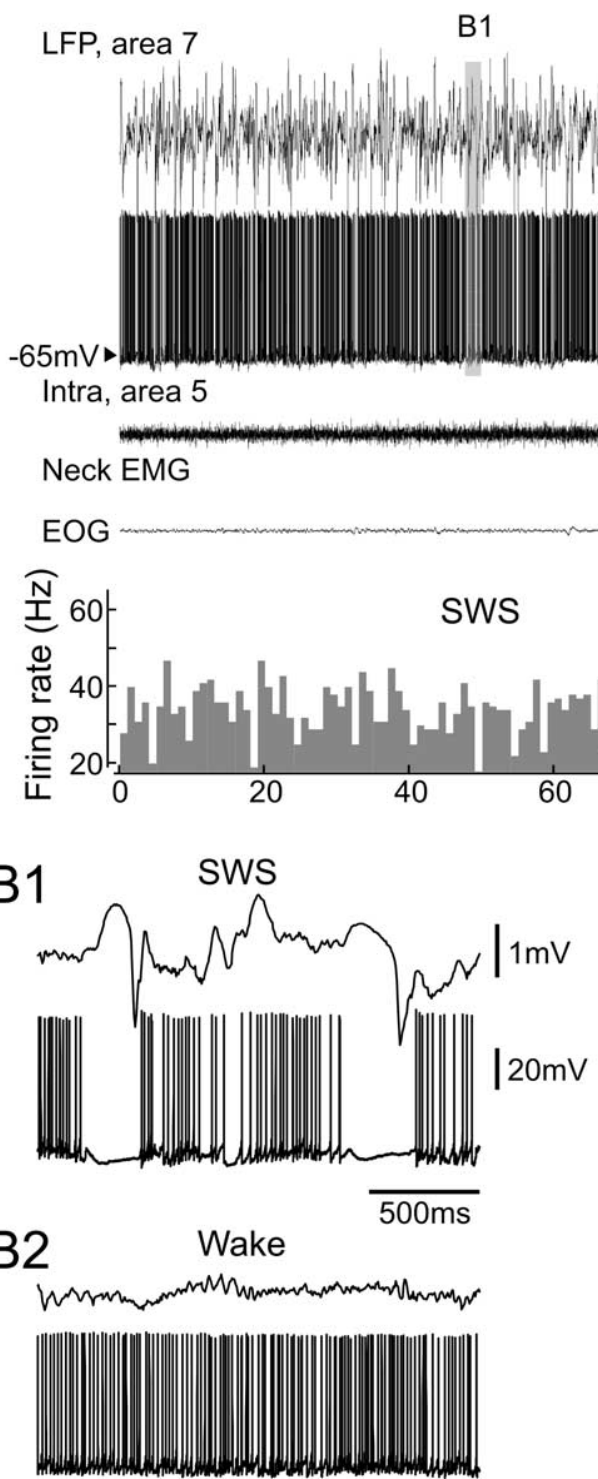

Wake

B2 $\quad 1 \mathrm{mV}$

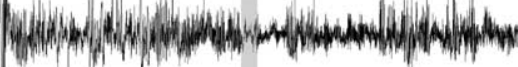

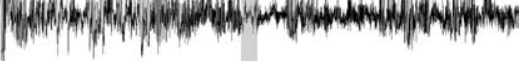

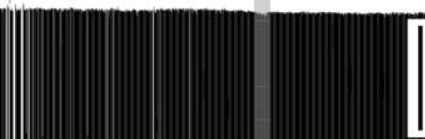
$50 \mathrm{mV}$

$\overline{10 s}$

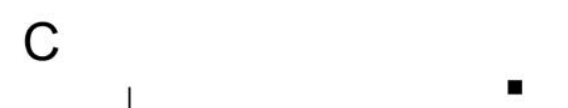

Figure 2. Activity of fast-spiking interneurons after awakening. $\boldsymbol{A}$, Intracellular activity of a fast-spiking neuron recorded simultaneously with LFPs, EMG, and EOG during the transition from slow-wave sleep to wake state. The onset of the waking state is indicated by the arrow. After awakening, the mean firing rate initially remained the same as during sleep (for $\sim 20 \mathrm{~s}$ ), then slightly increased (bottom, firing rate histogram). $\boldsymbol{B}$, Fragments of LFP and neuronal activities during slow-wave sleep and waking states are expanded as indicated in $\boldsymbol{A}$ by B1 and B2. C, Comparison of firing rates of regular-spiking and fast-spiking neurons in wake states. Pooled results showing the mean firing rate of $\mathrm{RS}$ (open circles) and FS (filled squares) represented against the mean $V_{\mathrm{m}}$ during waking.

active" cells in which the firing was sustained all through the wake state, as described previously (Matsumura et al., 1988; Baranyi et al., 1993; Steriade et al., 2001; Timofeev et al., 2001). In these wake-active neurons (Fig. 1A), the $V_{\mathrm{m}}$ was depolarized (around $-65 \mathrm{mV}$ ) and showed high-amplitude fluctuations and sustained irregular firing $(3.1 \mathrm{~Hz}$ on average; range, $1-11 \mathrm{~Hz})$ during wakefulness. During slow-wave sleep, all neurons always showed up and down states in the $V_{\mathrm{m}}$ activity in phase with the slow-waves (Fig. 1A, SWS), as described previously (Steriade et al., 2001).

Almost half of the RS neurons recorded (21 of 47), were "wake-silent" cells, which systematically ceased firing during periods of quiet wakefulness (Fig. $1 B$ ). Note that during the transition from slow-wave sleep to waking, these wake-silent neurons continued to fire for $10-60 \mathrm{~s}$, and after that period, their $V_{\mathrm{m}}$ hyperpolarized by several millivolts and they stopped to fire action potentials as long as the animal remained in the state of quiet wakefulness. Figure $1 B$ illustrates one example of a wake-silent cell that, after awakening, had a $V_{\mathrm{m}}$ of $-53.0 \pm 4.9 \mathrm{mV}$ and fired with frequency $10.1 \pm 7.9 \mathrm{~Hz}$ for $\sim 30 \mathrm{~s}$. Thereafter, the $V_{\mathrm{m}}$ hyperpolarized to $-62.5 \pm 2.6 \mathrm{mV}$ and the same neuron stopped firing. The hyperpolarization during waking state was not caused by $\mathrm{K}^{+}$ load because on two occasions we were able to obtain intracellular recordings from wake-silent neurons during waking state that was preceded and followed by other states of vigilance (supplemental Fig. 1 , available at www.jneurosci.org as supplemental material). In this case, the recorded neuron was relatively depolarized and fired action potentials during REM sleep. After awakening, this neuron was hyperpolarized by $\sim 10 \mathrm{mV}$ and stopped firing. After 3 min of waking state, the animal went to slow-wave sleep state and the same neuron was depolarized and started to fire action potentials. On one occasion (data not shown), we recorded extracellularly spikes from two units. One of the units stopped firing during waking state lasting $\sim 10 \mathrm{~min}$ whereas another unit continued to emit action potentials. This observation suggests that it is a particular set of neurons and not local networks that stop firing during quiet wakefulness. The mean firing rates for RS neurons were $6.1 \pm 6.7 \mathrm{~Hz}$ (silent neurons included; $10.1 \pm 5.6 \mathrm{~Hz}$ with silent neurons excluded). No wake-silent cells were observed for other neuronal classes than RS cells and, all together, wake-silent neurons represented $\sim 25 \%$ of the total number of recorded cells in the wake state. This large proportion of wake-silent neurons constitutes a first hint for an important role for inhibitory conductances during waking.

In contrast, no silent neuron was found for presumed interneurons. During quiet wakefulness, 22 neurons were electrophysiologically identified as fast-spiking (FS). They displayed virtually no adaptation and had action potential width of $0.27 \pm 0.08 \mathrm{~ms}$ (range, $0.15-0.45 \mathrm{~ms}$ ). After awakening, FS neurons tended to increase firing (Fig. $2 A, B$ ), and none of them was found to cease firing $(n=9)$. Interestingly, the increase of firing of FS neurons seems to follow the steady hyperpolarization of RS wake-silent neurons (Fig. $2 \mathrm{~A}$ ). The mean firing frequency of FS neurons was $28.8 \pm 20.4 \mathrm{~Hz}$ (range, $1-88 \mathrm{~Hz}$; only two neurons fired with frequency $<2 \mathrm{~Hz}$ ), which was significantly higher than that of RS neurons ( $p \leq 0.001$ ) (Fig. 2C). The mean $V_{\mathrm{m}}$ of FS neurons was $-61.3 \pm 4.5 \mathrm{mV}$, which is not significantly different from the $V_{\mathrm{m}}$ of RS neurons $(p=0.059)$.

To check for the contribution of $\mathrm{K}^{+}$conductances during quiet wakefulness, we recorded activities of three RS neurons with $\mathrm{Cs}^{+}$-filled pipettes (data not shown). The presence of ce- 
sium greatly affected the repolarizing phase of action potentials, demonstrating that $\mathrm{Cs}^{+}$was effective in blocking $\mathrm{K}^{+}$conductances, but the $V_{\mathrm{m}}$ distribution was marginally affected by the presence of cesium. The action of intracellular $\mathrm{Cs}^{+}$may overlap with the blocking action of neuromodulators on other $\mathrm{K}^{+}$conductances (McCormick, 1992; Metherate and Ashe, 1993), which may explain the absence of effect of $\mathrm{Cs}^{+}$on the $V_{\mathrm{m}}$. This preliminary evidence for a limited effect of cesium during wakefulness indicates that leak and $\mathrm{K}^{+}$conductances have no major effect on the $V_{\mathrm{m}}$ distribution, suggesting that it is mainly determined by synaptic conductances.

\section{Synaptic conductances in wakefulness and natural sleep}

To determine the relative contribution of excitatory and inhibitory conductances, we have analyzed the intracellular recordings described above using the previously proposed $\mathrm{VmD}$ method (Rudolph et al., 2004). This method assumes that the $V_{\mathrm{m}}$ fluctuations are caused by the combined action of two synaptic conductances, excitatory $\left(g_{e}\right)$ and inhibitory $\left(g_{i}\right)$ (see Materials and Methods). Instead of modeling explicitly the activity of thousands of synapses, one considers the $V_{\mathrm{m}}$ and synaptic conductances as stochastic variables, which are described by probability densities. Using this formalism, it is possible to obtain an analytic expression for the steady-state probability distribution of the $V_{\mathrm{m}}$, which is expressed as a function of the synaptic conductance parameters (Rudolph and Destexhe, 2003, 2005). Because the steady-state $V_{\mathrm{m}}$ distribution is observable experimentally, fitting the analytic expression to experimental $V_{\mathrm{m}}$ distributions provides estimates of the underlying synaptic conductances. This method was tested using dynamic-clamp methods and was shown to provide consistent estimates of synaptic conductances and their fluctuations (Rudolph et al., 2004).

We computed $V_{\mathrm{m}}$ distributions for periods of stationary activity during wakefulness and slow-wave sleep up states (SWSup). Figure $3 B$ (awake) shows $V_{\mathrm{m}}$ distributions of two different but representative cells obtained from periods of wakefulness in which the cat and the LFPs did not show any sign of drowsiness. The $V_{\mathrm{m}}$ distribution was approximately Gaussian, centered around $\bar{V}=-63.1 \mathrm{mV}$, and the SD of the $V_{\mathrm{m}}\left(\sigma_{V}\right)$ was $\sim 3.6 \mathrm{mV}$. During slow-wave sleep, we calculated the $V_{\mathrm{m}}$ distribution specifically during up states (Fig. 3B, SWS). It had an approximately similar shape as during wakefulness $\left(\bar{V}=-62.7 \mathrm{mV} ; \sigma_{V}=3.3\right.$ $\mathrm{mV})$. Similar distributions were also observed during REM sleep (data not shown). The $V_{\mathrm{m}}$ distributions were computed using several pairs of DC levels, which were selected in the linear portion of the $V-I$ relationship (Fig. $3 A$ ). The conductance estimates obtained for several of such pairings (see Materials and Methods) are represented in Figure 3C. In this example, during both wakefulness and slow-wave sleep up states, the inhibitory conductances were several-fold larger than excitatory conductances. Variations of different parameters, such as the leak conductances (Fig. 3D), or the parameters of synaptic conductances (see Materials and Methods), affected the absolute values of conductance estimates, but always pointed to the same qualitative effect of dominant inhibition. The sole exception was when considering high leak conductances, larger than the synaptic activity itself, in which case the excitatory and inhibitory conductance were of comparable magnitude (Fig. 3D, asterisk).

The VmD method also provides estimates of the variance of synaptic conductances. Similar to absolute conductance estimates, conductance variances were generally larger for inhibition (Fig. 3C). However, in contrast to absolute conductance estimates, the estimates of conductance variance do not depend on
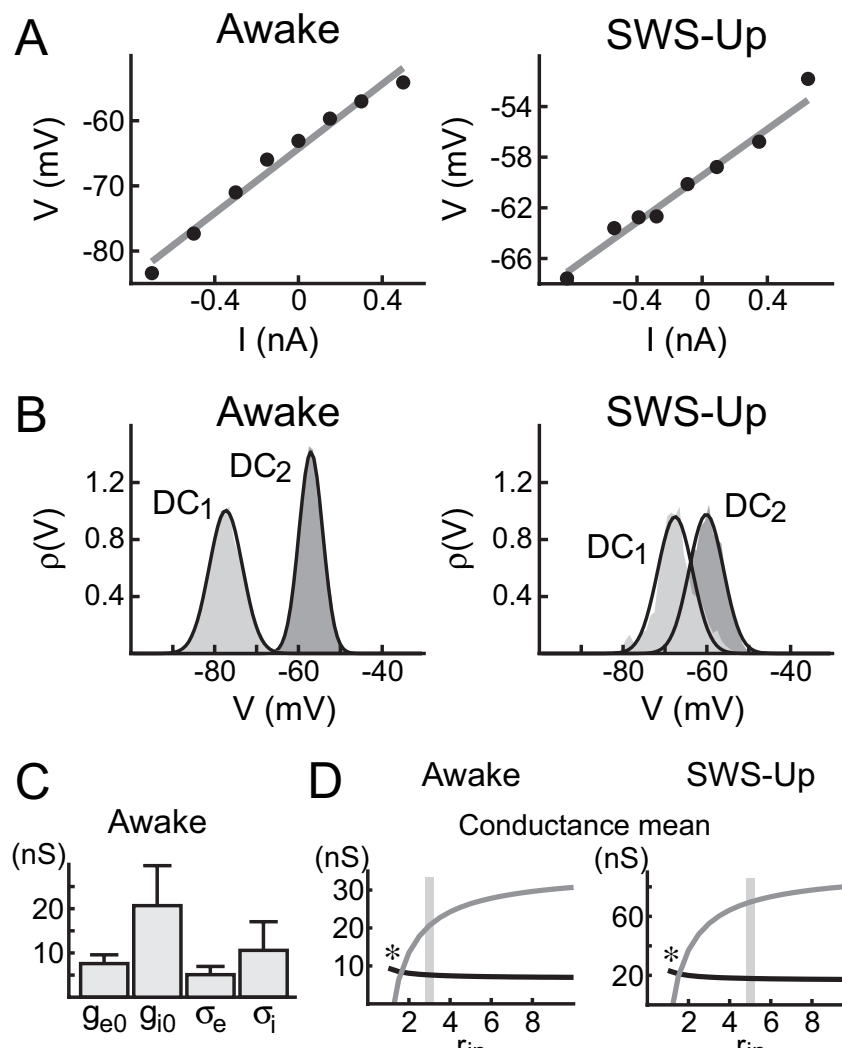

D Awake

SWS-Up
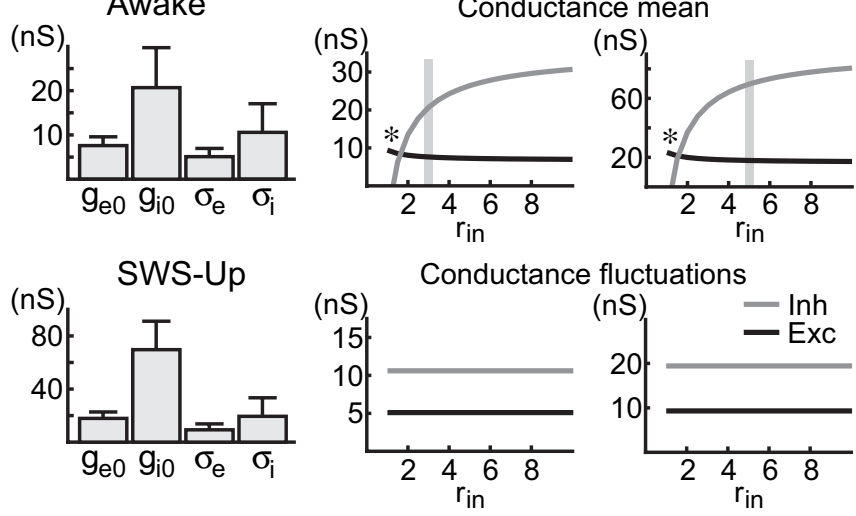

Figure 3. Estimation of conductances from intracellular recordings in awake and naturally sleeping cats. A, Voltage- current relationships obtained in two different cells during wakefulness (awake) and SWS-up. The average subthreshold voltage (after removing spikes) is plotted against the value of the holding current. $\boldsymbol{B}$, Examples of $V_{\mathrm{m}}$ distributions $\rho(\boldsymbol{V})$ obtained in the same neurons as in $\boldsymbol{A}$. The continuous lines show Gaussian fits of the experimental distributions. C, Conductance values (mean and SD) estimated by decomposing synaptic activity into excitatory and inhibitory components using the $V m D$ method (applied to 28 and 26 pairings of $V_{m}$ recordings at different $D$ C levels for awake and SWS up states, respectively). $D$, Variations of the value for conductance mean (top) and conductance fluctuations (bottom) as a function of different choices for the leak conductance. $r_{\text {in }}=R_{\text {in }}$ (quiescent) $/ R_{\text {in }}$ (active); asterisks indicate the region with high leak conductances in which excitation is larger than inhibition; the gray area shows the $r_{\text {in }}$ values used for the conductance estimates in $C$. Error bars indicate SD.

the particular choice of leak conductances (Fig. 3D, bottom). These results suggest that inhibition provides a major contribution to the $V_{\mathrm{m}}$ fluctuations.

This pattern was observed in the majority of cells analyzed, although a diversity of conductance combinations was present when considering the different states of vigilance, including periods of REM sleep. In cells for which synaptic conductances were estimated ( $n=11$ for awake, wake-active cells only; $n=7$ for SWS up states; $n=2$ for REM), the average $V_{\mathrm{m}}$ and fluctuation amplitude were comparable in all states [awake, $\bar{V}=-54.2 \pm 7.5$ $\mathrm{mV}, \sigma_{V}=2.4 \pm 0.7 \mathrm{mV}$; SWS-up, $\bar{V}=-58.3 \pm 4.9 \mathrm{mV}, \sigma_{V}=$ $2.7 \pm 0.5 \mathrm{mV}$; SWS down states (SWS-down), $\bar{V}=-67.0 \pm 6.9$ $\mathrm{mV}, \sigma_{V}=1.9 \pm 0.6 \mathrm{mV} ; \mathrm{REM}, \bar{V}=-58.5 \pm 5.2 \mathrm{mV}, \sigma_{V}=2.1 \pm$ $0.9 \mathrm{mV}$ ] (Fig. 4A). However, the total input resistance showed important variations (awake, $16.1 \pm 14.5 \mathrm{M} \Omega$; SWS-up, $12.3 \pm$ 
A

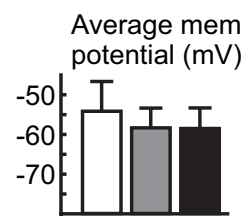

B

Conductance mean

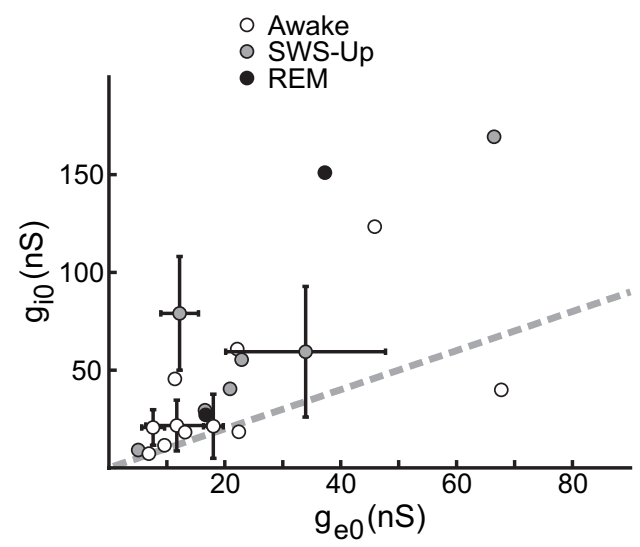
fluctuations (mV)

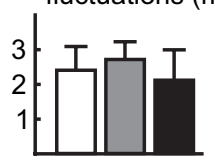

Membrane potential

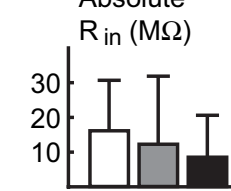

C
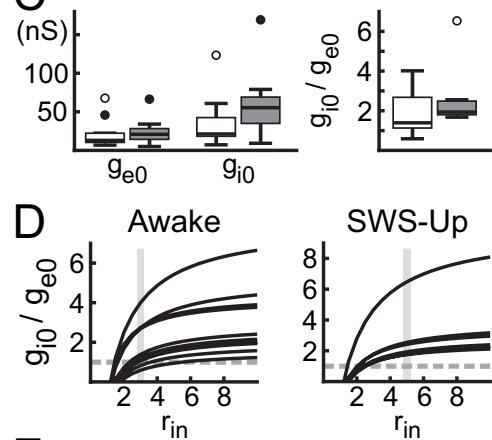

E

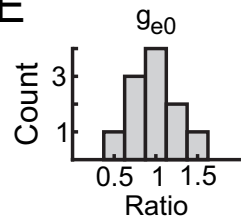

$\square$ Awake SWS-Up REM

Figure 4. Conductance estimates in cortical neurons during wake and sleep states. $\boldsymbol{A}$, Average $V_{\mathrm{m}}, V_{\mathrm{m}}$ fluctuation amplitude, and absolute input resistance $R_{\text {in }}$ during wakefulness (awake), SWS-up, and REM sleep periods, computed from all cells for which synaptic conductances were estimated. $\boldsymbol{B}$, Spread of excitatory $\left(g_{e 0}\right)$ and inhibitory $\left(g_{i 0}\right)$ conductance mean during wakefulness and slow-wave sleep up states. Estimated conductance values show a high variability among the investigated cells, but in almost all states, a dominance of inhibition was observed. $\boldsymbol{C}$, Box plots of mean excitatory and inhibitory conductance estimates (left) and average ratio between inhibitory and excitatory mean (right) observed during wakefulness and slow-wave sleep up states for the population shown in $\boldsymbol{B}$. In both states, dominant inhibition was observed, an effect that was more pronounced during SWS-up. $\boldsymbol{D}$ Variations of the ratio between inhibitory and excitatory mean conductance values as a function of different choices for the leak conductance. $r_{\text {in }}=R_{\text {in }}$ (quiescent) $/ R_{\text {in }}$ (active); the gray area indicates the values used for conductance estimation plotted in $B$ and $\boldsymbol{C}$. $\boldsymbol{E}$, Histograms of conductance values relative to the leak conductance during the wake state. Error bars indicate SD.

A

Conductance fluctuations
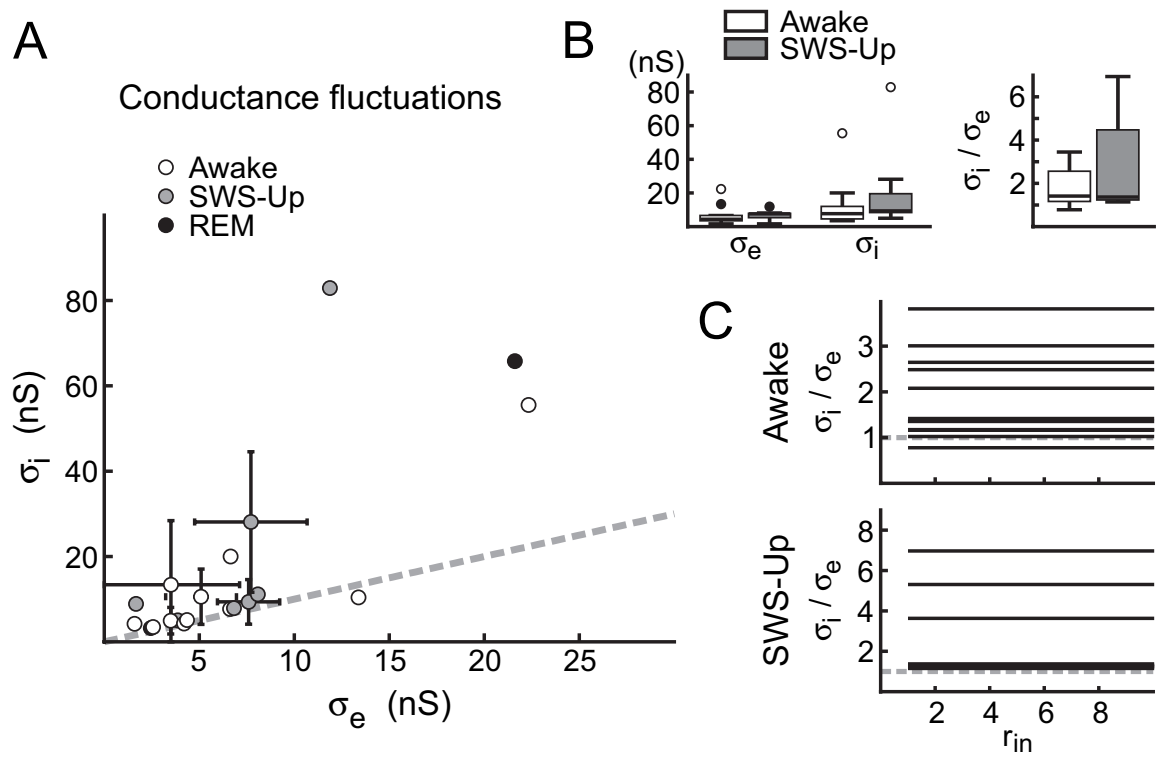

Figure 5. Estimates of conductance fluctuations from cortical neurons during wake and sleep states. $\boldsymbol{A}$, Spread of excitatory $\left(\sigma_{e}\right)$ and inhibitory $\left(\sigma_{i}\right)$ conductance fluctuations during wakefulness and slow-wave sleep up states. Estimated conductance values show a high variability among the investigated cells, but in all states a dominance of inhibition was observed. $\boldsymbol{B}$, Box plots of excitatory and inhibitory conductance fluctuation amplitude (left) and average ratio between inhibitory and excitatory SD (right) observed during wakefulness (awake) and SWS-up for population shown in $\boldsymbol{A}$. In all cases, dominant inhibition was observed. $C$, In the VmD method, estimated values for the ratio between inhibitory and excitatory conductance fluctuations do not depend on different choices for the leak conductance. $r_{\text {in }}=R_{\text {in }}$ (quiescent) $/ R_{\text {in }}$ (active). Error bars indicate SD.

19.6 M $\Omega$; SWS-down, $22.4 \pm 31.7 \mathrm{M} \Omega$; REM, $8.5 \pm 12.1 \mathrm{M} \Omega$ ), possibly caused by differences in the passive properties and cellular morphologies. The estimated synaptic conductances spread over a large range of values for both mean (ranging from 5 to $70 \mathrm{nS}$ and 5 to $170 \mathrm{nS}$ for excitation and inhibition, respectively; medians: 21 and $55 \mathrm{nS}$ for excitation and inhibition during SWS-up, 13 and $21 \mathrm{nS}$ for excitation and inhibition for awake, respectively) (Fig. $4 B, C$ ) and SD (ranging from 1.5 to $22 \mathrm{nS}$ and 3.5 to $83 \mathrm{nS}$ for excitation and inhibition, respectively; medians: 7.6 and $9.3 \mathrm{nS}$ for excitation and inhibition for SWS-up, 4.3 and $7.7 \mathrm{nS}$ for excitation and inhibition for awake, respectively) (Fig. $5 A, B)$. In all states and for reasonable assumptions for the leak conductance (Fig. $4 D$, gray), dominant inhibition was found in more than half of the cells analyzed $(n=$ 6 for awake and $n=7$ for SWS-up had $>40 \%$ larger mean inhibitory conductance; $n=6$ for awake and $n=4$ for SWS-up had $>40 \%$ larger inhibitory SD). In the remaining cells, inhibitory and excitatory conductance values were of comparable magnitude, with a tendency for a slight dominance of inhibition (except for $n=2$ cells in awake). Moreover, in all cells analyzed, inhibition was more pronounced during the up states of SWS (estimated ratios between inhibition and excitation were $2.7 \pm 1.4$ and $3.0 \pm 2.2$ for conductance mean and SD; medians were 1.9 and 1.4, respectively) compared with wakefulness (ratios of $1.8 \pm 1.1$ and $1.9 \pm$ 0.9 for conductance mean and SD; medians were 1.4 and 1.4, respectively) (Figs. $4 C, 5 B)$. Renormalizing the conductance values to the leak conductance for each cell in the wake state led to values that were more homogeneous (Fig. 4E). In this case, the excitatory conductance was of the order of the leak conductance (Fig. $4 E$, left) $(0.81 \pm 0.26)$, whereas inhibition was $\sim 1.5$ times larger (Fig. $4 E$, right) (1.26 \pm 0.31 ).

These results were also checked using the classic "ohmic" conductance analysis (see supplemental Methods, available at www.jneurosci.org as supplemental material). By integrating the $V_{\mathrm{m}}$ measurements in the various active states into the membrane equation (Eq. 8, supplemental Materials and Methods, available at www. jneurosci.org as supplemental material), we obtained estimates for the ratio between mean inhibitory (excitatory) conductances and the leak conductance for each cell (supplemental Fig. $2 A$, available at www.jneurosci.org as supplemental material). This and the pooled results 
for all available cells (supplemental Fig. $2 B$, available at www. jneurosci.org as supplemental material) also indicate that the relative contribution of inhibition is several-fold larger than that of excitation for both wakefulness and SWS up states. Average values are $\bar{g}_{i} / \bar{g}_{e}=3.2 \pm 1.3$ for SWS-up and $\bar{g}_{i} / \bar{g}_{e}=1.7 \pm 1.1$ during wakefulness. Here also, these values were relatively robust against the choice of the leak conductance (supplemental Fig. 2C, available at www.jneurosci.org as supplemental material).

In a small subset of cells $(n=3)$, the recording was long enough to span across several wake and sleep states so that SWS and wakefulness could be directly compared. In agreement with the reduction of the average firing rate of RS neurons during the transition from SWS to wakefulness, we observed a reduction of the mean excitatory conductance (values during wakefulness were between 40 and $93 \%$ of those during SWS-up) and its fluctuation amplitude (between 45 and $85 \%$ of those observed during SWS-up). In contrast to the observed increase of the firing rate of interneurons during sleep-wake transitions, the inhibitory conductances also decreased markedly (values during wakefulness were between 35 and $60 \%$ for the mean conductance, and between 10 and $71 \%$ for the SD compared with corresponding values during SWS-up) (see Discussion).

\section{Conductance time course during up- and down-state transitions}

We next estimated the time evolution of conductances during up and down states of slow-wave sleep. We performed a similar analysis as above, with the difference that $V_{\mathrm{m}}$ distributions were not calculated by accumulating statistics only over time, but also over repeated trials. Several up states $(n=1$, between 6 and 36 slow-wave oscillation cycles at eight DC levels) were selected and aligned with respect to the "down-up" transition as determined by the sharp LFP negativity (Fig. 6, left). The $V_{\mathrm{m}}$ distributions were then calculated within small $(10 \mathrm{~ms})$ windows before and after the transition. This procedure led to estimates of the time course of the conductances and their variances, as a function of time during "down-up" state transitions, and similarly for "updown" transitions (Fig. 6, right). It is important to note that conductance changes were estimated here relative to the down state, and not with respect to rest, as above. This analysis showed that, for this particular cell, the onset of the up state is driven by excitation, whereas inhibitory conductances activate with a delay of $\sim 20 \mathrm{~ms}$, after which they tend to dominate over excitation. Note that in this case, inhibition is only slightly larger than excitation, presumably because the reference state is here the down state, which does not represent the true resting state. In this cell, the end of the up state was preceded by a drop of inhibition (Fig. $6 B$, asterisk). The variance of inhibitory conductances was always larger than that of excitatory conductances (Fig. $6 \mathrm{~B}$, bottom).

\section{Dynamics of spike initiation during activated states}

To investigate how excitatory and inhibitory conductance dynamics affect spike initiation, we first simulated the above results using computational models. We used a spiking model with stochastic conductances (Eq. 2) (see Materials and Methods) whose parameters are given by the above estimates. Integrating the particular values of conductances shown in Figure $3 C$ led the model to generate $V_{\mathrm{m}}$ activity in excellent agreement with the intracellular recordings (Fig. $7 \mathrm{~A}, \mathrm{C}$, awake). All of the present conductance measurements during the waking state were simulated in a similar way and yielded $V_{\mathrm{m}}$ activity consistent with the recordings (two more examples with clearly dominant excitation or inhibition are shown in supplemental Fig. 3, available at www.jneurosci.org
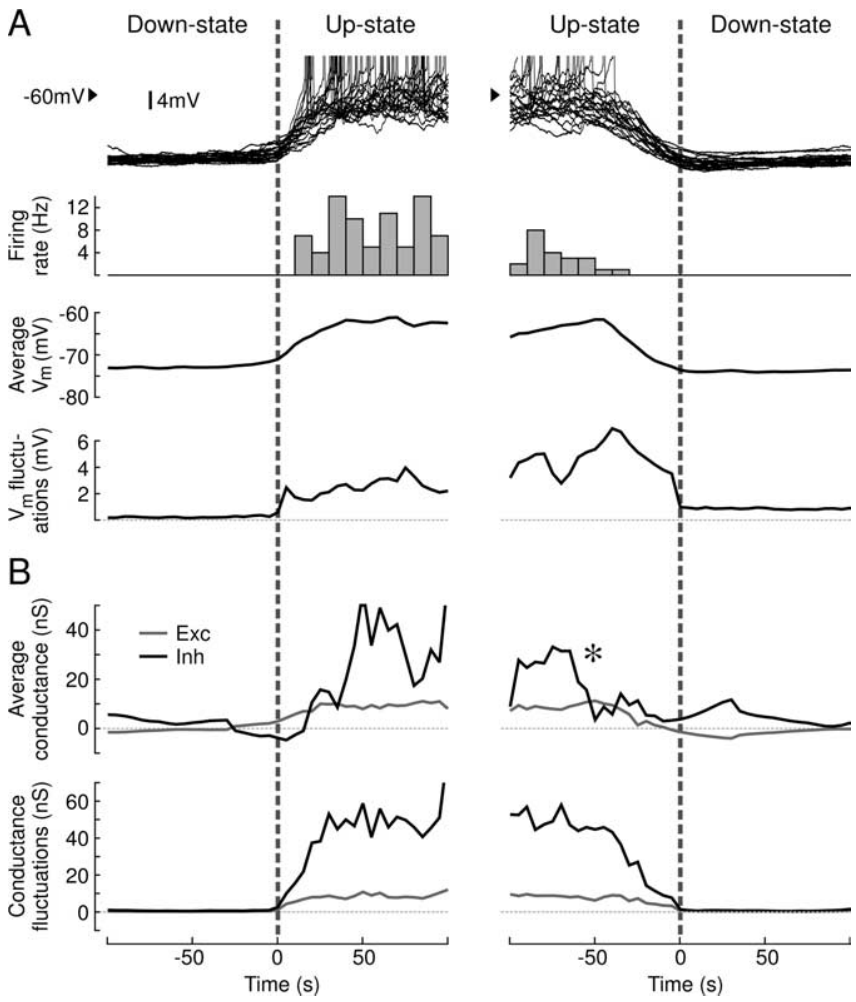

Figure 6. Conductance time course during up and down states during slow-wave sleep. $\boldsymbol{A}$, Superimposed intracellular traces during transitions from down to up states (left), and up to down states (right). $\boldsymbol{B}$, Time course of global synaptic conductances during down-up and up- down transitions. Conductance changes were evaluated relative to the average conductance of the down state. Top, Excitatory ( $g_{e}$, gray) and inhibitory $\left(g_{i}\right.$, black) conductances; asterisk indicates a drop of inhibitory conductance before the up- down transition. Bottom, SD of the conductances for excitation ( $\sigma_{e}$, gray) and inhibition $\left(\sigma_{i}\right.$, black). Both are shown at the same time reference as for $\boldsymbol{A}$.

as supplemental material). Similarly, integrating the conductance variations, given in Figure $6 \mathrm{~B}$, generated $V_{\mathrm{m}}$ activity consistent with the up-down state transitions seen experimentally (Fig. 7 B, C, SWS, SWS-up). These results show that the conductance estimates obtained above are consistent with the $V_{\mathrm{m}}$ activity recorded experimentally.

We next evaluated the optimal conductance changes related to spike initiation in the simulated wake state. Figure $8 \mathrm{~A}$ shows that the STA shows opposite variations for excitatory and inhibitory conductances preceding the spike. As expected, spikes were correlated to an increase of excitation (Fig. 8, excitatory). Less expected was that spikes were also correlated with a decrease of inhibitory conductance (Fig. 8 , inhibitory), so that the total synaptic conductance decreases before the spike (Fig. 8, total). Such a drop of total conductance was not present in simulated states when inhibition was not dominant (Fig. $8 \mathrm{~B}$ ). These results were checked using different combinations of parameters, and a drop of total conductance was always associated with inhibitiondominant states, except when the variance of inhibition was very small (data not shown). Such a drop of total conductance before the spike therefore constitutes a good predictor for inhibitiondominant states, given that conductance fluctuations are roughly proportional to their means.

This prediction was tested using intracellular recordings of electrophysiologically identified RS cells. We performed STAs of the $V_{\mathrm{m}}$ during wakefulness and the up states of SWS (Fig. 9A, Avg $\left.V_{\mathrm{m}}\right)$. The corresponding STA conductances were estimated by 
A

Awake

Excitatory conductance (nS)

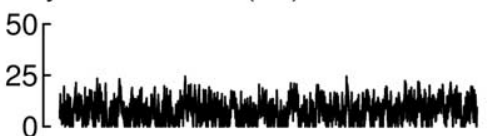

Inhibitory conductance (nS)

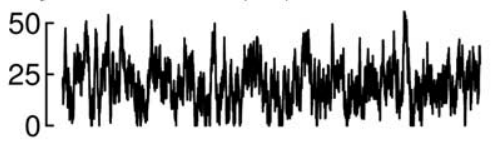

Membrane potential (mV)
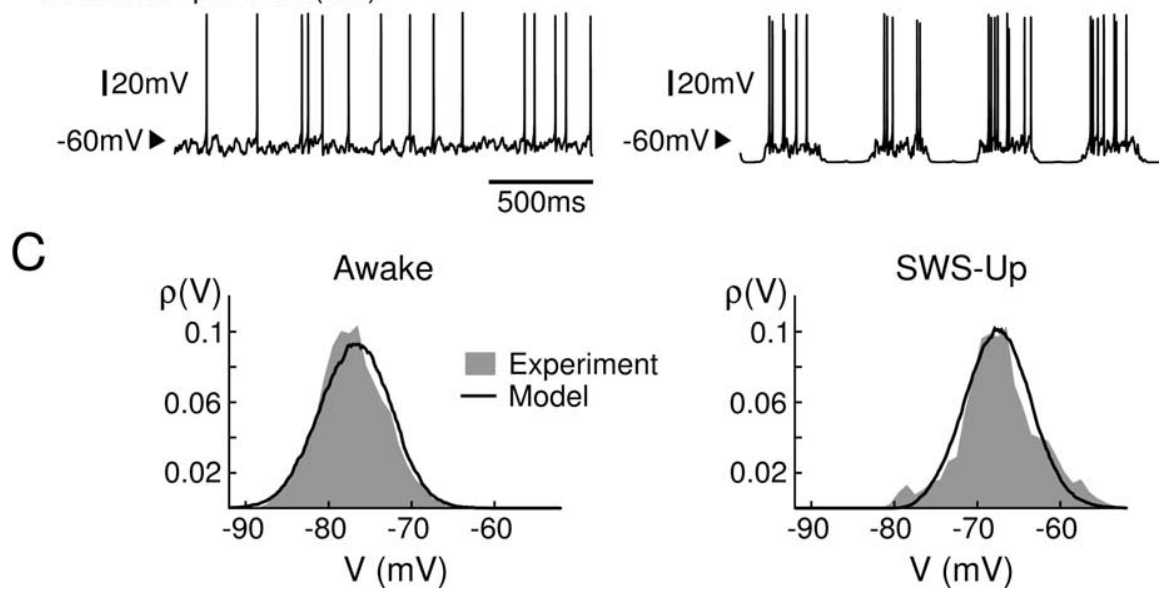

Figure 7. Model of conductance interplay during wakefulness and the up states of slow-wave sleep. $\boldsymbol{A}$, Simulated intracellular activity corresponding to measurements in the wake state (based on conductance values shown in Fig. 3C; leak conductance of $13.4 \mathrm{nS}$ ). $\boldsymbol{B}$, Simulated up and down state transitions (based on the values given in Fig. $6 B$ ). $C, V_{\mathrm{m}}$ distributions obtained in the model (solid lines) compared with that of the experiments (gray) in the same conditions ( $D C$ injection of -0.5 and $-0.43 \mathrm{nA}$, respectively).

discretizing the time axis and solving the membrane equation (see Materials and Methods). The STA conductances demonstrated a drop of total membrane conductance preceding the spike (Fig. $9 A$, total), which occurred on a similar time scale compared with the model (compare Fig. 8A). The decomposition of this conductance into excitatory and inhibitory components shows that the inhibitory conductance drops before the spike, whereas the excitatory conductance shows a steeper increase just before the spike (Fig. 9A) (note that the latter increase is probably contaminated by voltage-dependent currents associated with spike generation). Such a pattern was observed in most of the cells tested ( 7 of 10 cells in awake, six of six cells in SWS-up, and two of two cells in REM) (Fig. 9B,C). An example of a neuron that did not show such a drop of total conductance is given in supplemental Figure 4 (available at www. jneurosci.org as supplemental material). Most of the cells, however, yielded STAs qualitatively equivalent to that of the model when inhibition is dominant (Fig. $8 \mathrm{~A}$ ).

Finally, we investigated whether the dominance of inhibition (as deduced from conductance analysis) and the drop of conductance (from STA analysis) are related by including all cells for which both analyses could be done (Fig. 10). The total conductance change before the spike was clearly related to the difference of excitatory and inhibitory conductance deduced from $\mathrm{VmD}$ analysis (Fig. $10 \mathrm{~A}$, gray area), indicating that cells dominated by inhibition generally gave rise to a drop of total conductance before the spike. However, there was no quantitative relationship between the amplitude of those changes. Such a quantitative relationship was obtained for conductance fluctuations (Fig. $10 \mathrm{~B}$ ), which indicates that the magnitude and sign of the conductance change before the spike is strongly related to the relative amount of excitatory and inhibitory conductance fluctuations. The clear correlation between the results of these two independent analyses therefore confirms that most neurons have strong and highly fluctuating inhibitory conductances during wake and sleep states.

\section{Discussion}

We presented here a combination of intracellular recordings and computational models of cortical neurons that points to the following conclusions: (1) $V_{\mathrm{m}}$ activity during wake and sleep states results from diverse combinations of excitatory and inhibitory conductances, with dominant inhibition in most of the cases; (2) inhibitory conductance fluctuations are generally larger than for excitation; (3) spike initiation is in most cases correlated with a decrease of inhibition, which appears as a transient drop of membrane conductance before the spike. We discuss below the significance of these findings.

It is important to note that the excitatory and inhibitory conductances were estimated here from somatic recordings. The values obtained therefore reflect the overall conductances as seen from the soma, after dendritic integration, and are necessarily different from the "total" con-

ductance present in the soma and dendrites of the neuron. However, these somatic estimates are close to the conductance interplay underlying spike generation because the spike initiation zone (presumably in the axon) (Stuart et al., 1997) is electrotonically close to the soma.

Using such somatic estimates, dominant inhibition was seen in all cells during the up-states of slow-wave sleep $(n=7$ cells had $>40 \%$ larger inhibitory conductance), and in the majority of neurons recorded in the wake state $(n=6$ cells showed clear dominant inhibitory conductances). This conclusion is robust to a number of parameter variations, including different hypotheses about the leak conductance. A notable exception is when high leak conductances (larger than synaptic activity) are assumed, in which case excitatory and inhibitory conductances are of comparable magnitude. However, this possibility is very unlikely in cats because high leak conductances would predict dramatic effects of cesium on the $V_{\mathrm{m}}$ distribution, which is not what we observed (I. Timofeev, unpublished observations). Moreover, intracellular measurements in cats show that the total conductance resulting from intense synaptic activity is several-fold larger than the leak conductance (Borg-Graham et al., 1998; Paré et al., 1998). Nevertheless, some neurons were found to have roughly equal excitatory and inhibitory conductances during waking for reasonable values of the leak conductance (Fig. $4 D$ ). These results suggest that there exists a diversity of combinations of excitation and inhibition, which varies from cell to cell in the same network, as 
also shown previously for visual responses in anesthetized cats (Monier et al., 2003).

However, with the exception of one cell, the variance of inhibition was always larger than that of excitation, for all parameters tested. In contrast to conductance estimates, the $\mathrm{VmD}$ estimate of conductance variance does not depend on any hypothesis about the leak conductance. This high level of inhibitory fluctuations is consistent with previous measurements in cortical neurons under anesthesia (Hasenstaub et al., 2005; Rudolph et al., 2005). It suggests that a major part of $V_{\mathrm{m}}$ fluctuations is caused by variations of inhibitory conductances. Because variations of $V_{\mathrm{m}}$ are most effective on evoking spikes, rather than the absolute $V_{\mathrm{m}}$ level (Mainen and Sejnowski, 1995), these results suggest that inhibition is particularly effective in evoking spikes (see below).

Interestingly, we also found that a significant proportion of excitatory neurons cease firing during the wake state. Such wake-silent cells were also observed in motor cortex of young rats using whole-cell recordings (Brecht et al., 2004). The high proportion of these wake-silent cells (44.6\%) suggests that there should be less excitatory conductance during wakefulness compared with slow-wave sleep, which is indeed what we observed. Interneurons also show diverse behaviors: the majority of FS cells recorded show an increase of firing in the wake state, whereas a minority shows the opposite (data not shown). In contrast, all excitatory cells analyzed in the sleep-wake transition $(n=3)$ show a decrease of inhibition at the transition to waking. This discrepancy can be explained by bias in our sampling of different populations of interneurons, which are known to display opposite sensitivity to neuromodulators (Xiang et al., 1998), or by a different state of depression/potentiation at inhibitory synapses, depending on behavioral state. In agreement with a previous study under anesthesia (Rudolph et al., 2005), these differences also suggest that up states and EEG-activated states stem from similar, but nonidentical network states.

In contrast with the present results, previous conductance measurements showed that, paradoxically, up and down states have a comparable input resistance in rat barrel cortex in vivo (Zou et al., 2005; Waters and Helmchen, 2006). The difference is not caused by contamination by action-potential related $\mathrm{Na}^{+}$ currents, as suggested by Waters and Helmchen (2006), because the spikes are removed in our $\mathrm{VmD}$ analysis and the $\mathrm{V}-\mathrm{I}$ relationships were linear. Indeed, applying the $\mathrm{VmD}$ method to barrel cortex in vivo also gave the same paradoxical results (Zou et al., 2005), which were radically different from those in the association cortex of cats using the same method (Rudolph et al., 2005) (present study). High-conductance states have also been observed in vivo in cat visual cortex (Borg-Graham et al., 1998; Hirsch et al., 1998; Anderson et al., 2000), in cat association cortex (Paré et al., 1998; Rudolph et al., 2005), and in ferret visual cortex (Haider et al., 2006), which suggests fundamental differences of network dynamics in different cortical regions.
The present evidence for dominant inhibition also contrasts with the roughly equal conductances measured in voltage-clamp during spontaneous up-states in ferret cortical slices (Shu et al., 2003) or in vivo (Haider et al., 2006). Although we also observed neurons with roughly equal conductances $(n=5$ for wake, none for SWS-up), this does not explain the differences. A possible explanation is that those voltage-clamp measurements were performed in the presence of $\mathrm{Na}^{+}$and $\mathrm{K}^{+}$channel blockers (QX314 and cesium), and these drugs affect somatodendritic attenuation by reducing the resting conductance. Consequently, excitatory events located in dendrites have a more powerful impact on the soma compared with the intact neuron, which may explain the discrepancy. Another possible explanation is that, in voltageclamp experiments, when the voltage clamp is applied from the soma, the more distal regions of the cell are unlikely to be clamped, which may result in errors in estimating conductances and reversal potentials. Moreover, in this case, the presence of uncompensated electrode series resistance may worsen the estimates or affect the ratio between excitation and inhibition (for simulations, see supplemental Table 1, available at www.jneurosci.org as supplemental material). Additional conductance measurements should be performed in nonanesthetized animals to address these issues. However, our results are in agreement with conductance measurements performed in cortical neurons in vivo under anesthesia, which also show evidence for dominant inhibitory conductances (Borg-Graham et al., 1998; Hirsch et al., 1998; Destexhe et al., 2003; Rudolph et al., 2005).

Not only inhibition seems to provide a major contribution to the conductance state of the membrane, but also the conductance 

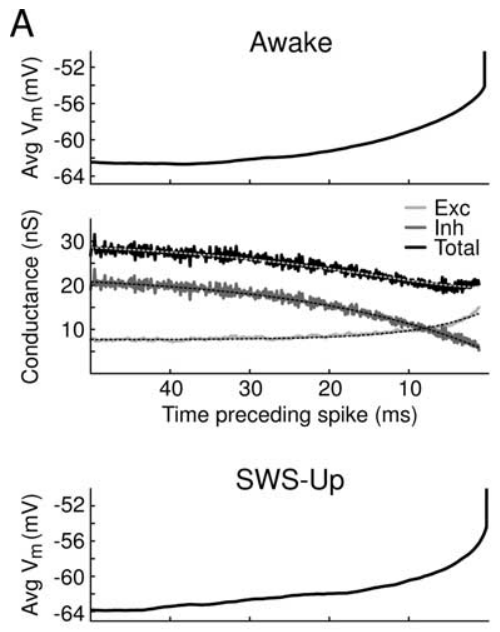

B
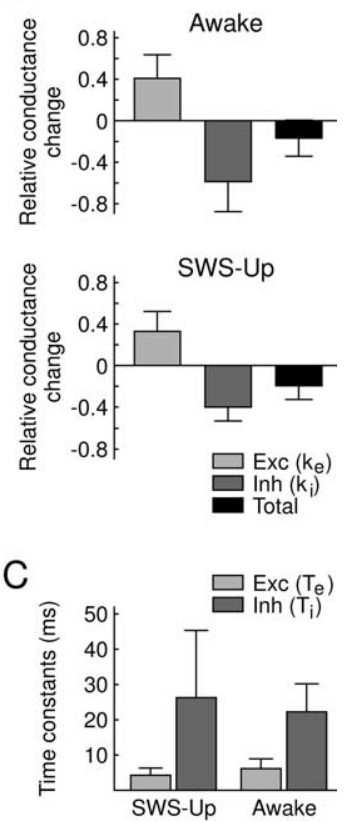

Figure 9. Decrease of membrane conductance preceding spikes in wake and sleep states. $\boldsymbol{A}$, STA for the membrane potential (Avg $V_{m}$ ) as well as excitatory (Exc), inhibitory (Inh), and total conductances obtained from intracellular data of regular-spiking neurons in an awake (top) and sleeping (SWS-up, bottom) cat. The estimated conductance time courses showed in both cases a drop of the total conductance caused by a marked drop of inhibitory conductance within $\sim 20$ $\mathrm{ms}$ before the spike. $\boldsymbol{B}$, Average value for the relative conductance change $\left(k_{e}\right.$ and $\left.k_{i}\right)$ triggering spikes during wakefulness (top) and up-states during SWS (bottom) obtained from exponential fits of the STA conductance time course (using Eq. 3) for all investigated cells. A decrease of the total membrane conductance and of the inhibitory conductance is correlated with spike generation, similar to the model (Fig. $8 A$ ) (estimated values: $k_{e}=0.41 \pm 0.23, k_{i}=-0.59 \pm 0.29$; total change, $-0.17 \pm 0.18$ for awake, $k_{e}=0.33 \pm 0.19, k_{i}=-0.40 \pm 0.13 ;-0.20 \pm$ 0.13 for SWS-up). C, Time constants of average excitatory and inhibitory conductance time course ahead of a spike in SWS and wake states (estimated values: $T_{e}=4.3 \pm 2.0 \mathrm{~ms}$, $T_{i}=26.3 \pm 19.0 \mathrm{~ms}$ for SWS; $T_{e}=6.2 \pm 2.8, T_{i}=22.3 \pm 7.9 \mathrm{~ms}$ for awake). Error bars indicate SD.
A

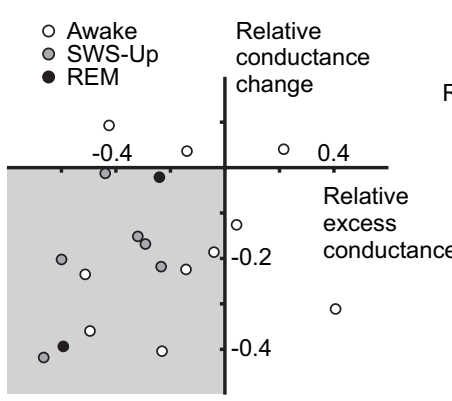

B

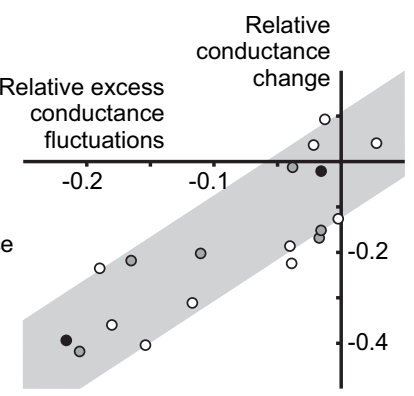

Figure 10. Relationship between conductance STA and the estimates of conductance and variances. $A$, Relationship between total membrane conductance change before the spike (relative conductance change; Eq. 4) obtained from STA analysis, and the difference of excitatory and inhibitory conductance (relative excess conductance; Eq. 5) estimated using the VmD method. Most cells are situated in the bottom-left quadrant (gray), indicating a relationship between inhibitory-dominant states and a drop of membrane conductance before the spike. $\boldsymbol{B}$, Relationship between relative conductance change before the spike and conductance fluctuations, expressed as the difference between excitatory and inhibitory fluctuations (relative excess conductance fluctuations; Eq. 6). Here, a clear correlation (gray area) shows that the magnitude of the conductance change before the spike is related to the amplitude of conductance fluctuations. Open circles, Wake; gray circles, SWS-up; black circles, REM. For definitions, see Materials and Methods. variations, which are larger for inhibition compared with excitation. This suggests that inhibition widely contributes to setting the $V_{\mathrm{m}}$ fluctuations and, therefore, it presumably has a strong influence on action-potential firing. This hypothesis was tested in computational models, which predicted that when inhibition is dominant, spikes are correlated with a previous decrease of inhibition, rather than an increase of excitation. This decrease of inhibition should be visible as a membrane conductance decrease before the spike, which is indeed what we observed in most neurons analyzed in wake and sleep states (Fig. 9). A prominent role for inhibition is also supported by previous intracellular recordings demonstrating a time locking of inhibitory events with action potentials in awake animals (Timofeev et al., 2001), and the powerful role of inhibitory fluctuations on spiking in anesthetized states (Hasenstaub et al., 2005). Together, these results suggest that strong inhibition is not a consequence of anesthesia, but rather represents a property generally seen in awake and natural sleep states, pleading for a powerful role for interneurons in determining neuronal selectivity and information processing.

\section{References}

Anderson JS, Carandini M, Ferster D (2000) Orientation tuning of input conductance, excitation and inhibition in cat primary visual cortex. J Neurophysiol 84:909-926.

Baranyi A, Szente MB, Woody CD (1993) Electrophysiological characterization of different types of neurons recorded in vivo in the motor cortex of the cat. II. Membrane parameters, action potentials, current-induced voltage responses and electrotonic structures. J Neurophysiol 69:1865-1879.

Borg-Graham LJ, Monier C, Frégnac Y (1998) Visual input evokes transient and strong shunting inhibition in visual cortical neurons. Nature 393:369-373.

Brecht M, Schneider M, Sakmann B, Margrie TW (2004) Whisker movements evoked by stimulation of single pyramidal cells in rat motor cortex. Nature 427:704-710.

Destexhe A, Paré D (1999) Impact of network activity on the integrative properties of neocortical pyramidal neurons in vivo. J Neurophysiol 81:1531-1547.

Destexhe A, Rudolph M, Fellous JM, Sejnowski TJ (2001) Fluctuating synaptic conductances recreate in vivo-like activity in neocortical neurons. Neuroscience 107:13-24.

Destexhe A, Rudolph M, Paré D (2003) The high-conductance state of neocortical neurons in vivo. Nat Rev Neurosci 4:739-751.

Destexhe A, Timofeev I, Rudolph M (2005) Combining stochastic models with intracellular recordings reveals dominant inhibitory conductances in cortical neurons of awake and naturally-sleeping animals. Paper presented at COSYNE Conference, Salt Lake City, March.

Haider B, Duque A, Hasenstaub AR, McCormick DA (2006) Neocortical network activity in vivo is generated through a dynamic balance of excitation and inhibition. J Neurosci 26:4535-4545.

Hasenstaub A, Shu Y, Haider B, Kraushaar U, Duque A, McCormick DA (2005) Inhibitory postsynaptic potentials carry synchronized frequency information in active cortical networks. Neuron 47:423-435.

Hines ML, Carnevale NT (1997) The Neuron simulation environment. Neural Comput 9:1179-1209.

Hirsch JA, Alonso JM, Clay Reid R, Martinez LM (1998) Synaptic integration in striate cortical simple cells. J Neurosci 18: 9517-9528.

Kuhn A, Aertsen A, Rotter S (2004) Neuronal integration of synaptic input in the fluctuation-driven regime. J Neurosci 24:2345-2356.

Mainen ZF, Sejnowski TJ (1995) Reliability of spike timing in neocortical neurons. Science 268:1503-1506.

Matsumura M, Cope T, Fetz EE (1988) Sustained excitatory synaptic input to motor cortex neurons in awake animals revealed by intracellular recording of membrane potentials. Exp Brain Res 70:463-469.

McCormick DA (1992) Neurotransmitter actions in the thalamus and cerebral cortex and their role in neuro-modulation of thalamocortical activity. Prog Neurobiol 39:337-388.

Metherate R, Ashe JH (1993) Ionic flux contributions to neocortical slow waves and nucleus basalis-mediated activation: whole-cell recordings in vivo. J Neurosci 13:5312-5323. 
Monier C, Chavane F, Baudot P, Graham LJ, Frégnac Y (2003) Orientation and direction selectivity of synaptic inputs in visual cortical neurons: a diversity of combinations produces spike tuning. Neuron 37:663-680.

Paré D, Shink E, Gaudreau H, Destexhe A, Lang EJ (1998) Impact of spontaneous synaptic activity on the resting properties of cat neocortical neurons in vivo. J Neurophysiol 79:1450-1460.

Pospischil M, Rudolph M, Shulz D, Timofeev I, Destexhe A (2005) Are we inhibited when we are awake? A combined intracellular and computational analysis of membrane potential dynamics in cortical neurons of awake and naturally sleeping animals. Soc Neurosci Abstr 31: 276.15.

Pospischil M, Piwkowska Z, Rudolph M, Bal T, Destexhe A (2007) Calculating event-triggered average synaptic conductances from the membrane potential. J Neurophysiol 97:2544-2552.

Press WH, Flannery BP, Teukolsky SA, Vetterling WT (1986) Numerical recipes. The art of scientific computing. Cambridge, MA: Cambridge UP.

Priebe NJ, Ferster D (2005) Direction selectivity of excitation and inhibition in simple cells of the cat primary visual cortex. Neuron 45:133-145.

Rudolph M, Destexhe A (2003) Characterization of subthreshold voltage fluctuations in neuronal membranes. Neural Comput 15:2577-2618.

Rudolph M, Destexhe A (2005) An extended analytic expression for the membrane potential distribution of conductance-based synaptic noise. Neural Comput 17:2301-2315.

Rudolph M, Destexhe A (2006) Multichannel shot noise approach to describe synaptic background activity in neurons. Eur Phys J B 52:125-132.

Rudolph M, Piwkowska Z, Badoual M, Bal T, Destexhe A (2004) A method to estimate synaptic conductances from membrane potential fluctuations. J Neurophysiol 91: 2884-2896.

Rudolph M, Pelletier JG, Paré D, Destexhe A (2005) Characterization of synaptic conductances and integrative properties during electricallyinduced EEG-activated states in neocortical neurons in vivo. J Neurophysiol 94: 2805-2821.

Shu Y, Hasenstaub A, McCormick DA (2003) Turning on and off recurrent balanced cortical activity. Nature 423:288-293.

Steriade M, Timofeev I, Grenier F (2001) Natural waking and sleep states: a view from inside neocortical neurons. J Neurophysiol 85:1969-1985.

Stuart G, Spruston N, Sakmann B, Hausser M (1997) Action potential initiation and backpropagation in neurons of the mammalian CNS. Trends Neurosci 20:125-131.

Timofeev I, Grenier F, Steriade M (2001) Disfacilitation and active inhibition in the neocortex during the natural sleep-wake cycle: an intracellular study. Proc Natl Acad Sci USA 98:1924-1929.

Wehr M,Zador AM (2003) Balanced inhibition underlies tuning and sharpens spike timing in auditory cortex. Nature 426:442-446.

Xiang Z, Huguenard JR, Prince DA (1998) Cholinergic switching within neocortical inhibitory networks. Science 281:985-988.

Waters J, Helmchen F (2006) Background synaptic activity is sparse in neocortex. J Neurosci 26:8267-8277.

Zou Q, Rudolph M, Roy N, Sanchez-Vives M, Contreras D, Destexhe A (2005) Reconstructing synaptic background activity from conductance measurements in vivo. Neurocomputing 65:673-678. 